\title{
sciendo
}

DOI: 10.1515/aon-2019-0009

\section{SEAMLESS NAVIGATION IN GPS-DENIED ENVIRONMENT USING LIRHOT'S REAL TIME NORTH FINDING SENSOR}

\author{
Shlomi Voro \\ Lirhot Systems Ltd \\ Rehovot Israel \\ info@lirhot.com \\ Itamar Lavidor \\ Lirhot Systems Ltd \\ Rehovot Israel \\ info@lirhot.com
}

\begin{abstract}
This paper presents a new type of north finding sensor. The passive optical sensor captures images of the sky at a high frame rate and analyzes them into a polarized map of the sky with a high degree of accuracy. The sensor operates in real time, under various weather and atmospheric conditions. The sensor output shows high heading accuracy relative to the celestial true north. Based upon the NAS-14V2 astronomical method of navigation, it is possible to define the sensor global position on earth.
\end{abstract}

Keywords - North finding; automated astro-navigation; automated stellar navigation systems; Sky polarization; imaging sensor; navigation by natural network; autonomous passive sextant

\section{INDRUCTION}

Decades before the advent of satellite navigation or a global navigation satellite system (GNSS), manual Astro-inertial Navigation System remained a skill used in both the maritime environment and in the skies. Even fairly modern aircraft were designed with this form of navigation in mind.

Today, GPS and other Global Navigation Satellite Systems (GNSS) are used in everything from cellular communication networks, to basic consumer goods, high-end military systems, and stock trading inputs. But these systems are vulnerable: by attacking positioning, navigational, and timing (PNT) data through electronic warfare (EW) capabilities, state and non-state actors can cause significant damage to major economies, and everyday consumers alike.

The optical passive sensor introduced here utilizes a scattering phenomenon that occurs in the atmosphere and underwater and can be seen via the space. The sensor able to navigate without the use of GNSS or any other methods of Radio Frequencies (RF).

The sensor is capable to compute its global position on earth with accuracy of $300 \mathrm{~m}-400 \mathrm{~m}$, since it is not depended on the celestial body actual size nor parallax correction is needed.

Beside computing its global position, Lirhot sensor defines its heading vector to the True-North in real-time. There are only 4 known types of navigational sensors. (1) magnetic based sensors are capable of measuring the heading vector to the Magnetic-North but these sensors are sensitive to local and global magnetic field interferences. In addition, the earth's magnetic field is deviating over time and in a random manner. (2) Gyroscopic north-seeking sensors can find the direction to the Gyroscopic-North. These sensors can measure 
the changes in the gravity vector, and by doing so, define the Gyroscopic-North with relatively high accuracy. Unfortunately, the accuracy of these gyro devices suffers from degradation over time, mainly due to drift. The Gyroscopic north-seeking devices are costly both in price and in integration time. (3) The popular gyroscopic sensors based on MEMS technology can also measure the angular rate. These sensors are widely used today, mainly because of their low cost and long-term availability. However, these MEMS Gyroscopic sensors are poor in maintaining the azimuth direction and have a high drift level. In most cases, mainly when at motionless state, these MEMS devices are unable to define the azimuth pointing to the Gyroscopic-North. (4) An alternative way to find the heading vector to the True-North is by inputting the position of celestial bodies. It is possible to navigate accurately using a sextant device that measures the angle position of the celestial bodies and by doing so accurately determines the azimuth pointing to the True-North. It is only method today suits to navigation in GPS-denied environment.

This paper sheds light on a new type of north-finding optical sensor based upon celestial navigation methods. This highly accurate, low cost passive optical sensor has a small footprint and is composed of an off-the-shelf image sensor and processing unit [1].

The celestial methods of Navigation are well known for hundreds of years. The new type of sensor introduced here operates with similar astronomical (astro) navigation method to the mechanical NAS-14V2 which has an excellent position accuracy [2].

Unlike the leveled NAS-14V2 with astronomical method of navigation which locks onto three brightest celestial bodies, and has an accuracy position on earth of $90 \mathrm{~m}$. This paper explains a motionless lock on a single body (the sun) without the use of any type of tilt sensor nor inertia measurement unit (IMU).

\section{Principles OF OPERATION}

\section{A. The sensing method}

By sensing the atmospheric light from any location on earth, even from the air downward or from underwater, the sensor can calculate its angle in relation to the celestial body (sun or moon). Since the location of the celestial body is known, the compass's heading towards the celestial true north could be defined.

The sensor operates as an autonomous sextant, sidestepping the need for an unobstructed sightline of the celestial body. The sensor provides an azimuth heading and a gravity vector sensed by all 3 angles: roll, pitch and yaw (heading to the celestial true north). Includes its global position on earth.

\section{B. Atromsphric scattring effect}

It is well-known that the sky dome is polarized under various atmospheric conditions. The light polarization of the sky dome is a result of the Rayleigh Scattering Effect [2]. And it occurs when the celestial body (Sun or Moon) rays interact with the molecules and other particles in the atmosphere depth. As a result, the degree and angle of polarization are not uniform across the sky dome. Hence, the classic Scattering Theory claims the existence of a well-known angle ratio between the observation point and the sun's position in the sky [4]. It also highlights the angular distance between the global position of the observer and the celestial body (Sun or Moon) position in the sky.

It is possible to observe the skylight polarization and accurately detect the azimuth heading to the sun by attaching a rotating linearly polarizing filter in front of the camera [5] [6]. This method requires moving parts, it takes considerable time to compute and it is not sensitive enough to accurately analyze the azimuth pointing to the sun. Moreover, as this method takes time to complete, it tends to suffer from disturbances from adverse atmospheric conditions such as clouds, haze, fog and other environmental conditions that change over time.

\section{Getting azimuth heading using skylight polarization}

An innovative approach to measuring the direction to a celestial body using a passive optical path was suggested and demonstrated first by [1]. The new approach includes a designed polarizing filter that covers 
various polarization angles. This filter allows us to create an image of the polarization map of the sky at a snap. Our designed proprietary filter detects the direction to the Sun with high accuracy. The filter allows us to transform the polarized map of the sky into a single image in the shape of "butterfly" unlike array of photodiodes method [7]. Hence, the angular position of the "butterfly" wings highlights the direction to the celestial body. In this case, the wings indicate the direction to the position of the celestial body. Below in figure 1, we present an images captured by our sensor.

Fig. 1. 600pix by 400 pix image, the upper $1 / 2$ of the image shows the "butterfly" wings (in false colors) representing the polarization map of the sky. The $2^{\text {nd }}$ half of the image represents the diraction of driving. The system has sampling rate of $60 \mathrm{~Hz}, 0.16 \mathrm{degree}$ to

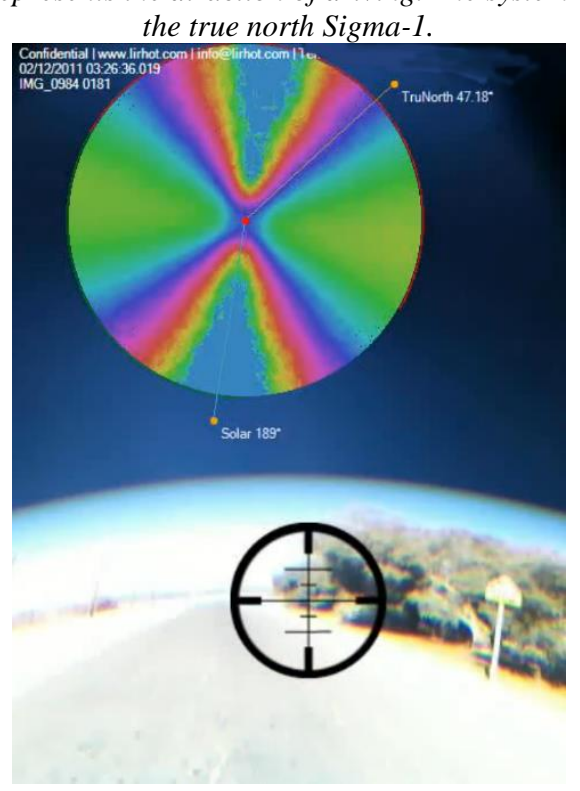

III. MAIN RESUlST

Lirhot sensor has placed in a tilted position and at a motionless state during a clear day for 5 hours on Tue.15th January 2019. The sensor kept its UTC time while capturing every second an image of the sky. The sensor was stood with unknown tilt and with unknown azimuth heading to the True North. The sensor location was $31^{\circ} 54^{\prime} 43.4^{\prime \prime} \mathrm{N} 34^{\circ} 48^{\prime} 24.6^{\prime \prime} \mathrm{E}$.

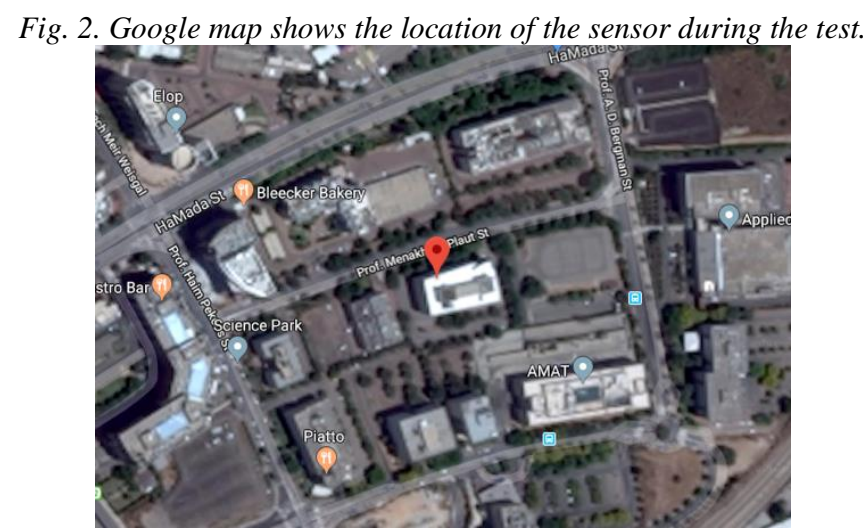

During the test, the sensor collected 18k samples in total, which sample represented by an image captured by the sensor. The sensor has computed each image into a polarization map of the sky. This computational process defines the azimuth angle between the sensor optical axis and the location of the Sun on the North East Down (NED) coordinates also known as Local tangent plane coordinates. The location of the Sun has been measured by 2 angles, Azimuth and Elevation. 


\section{A. Finding the bearings of the sensor optical axis}

Since the UTC time was known when each image was taken, it was possible numerically to find the bearings of the sensor's optical axis. By using all the $18 \mathrm{k}$ samples, it was possible to establish the sensor roll, pitch and azimuth heading to the true north.

The numerical search could be achieved by the fact that the sensor was in a motionless state, and so its azimuth heading should be kept at a constant value all along the $5 \mathrm{hrs}$ test duration.

Fig. 3. A graph represents 5 hrs of motionless state. During this time (x-axis) the sun has diped in elevation by 20 degrees (blue plot) and the theortical azimuth of the sun has shifted by 78.12 degrees in total, from 159.04deg @ 10:33:36 to 237.16deg @ 15:21:36.

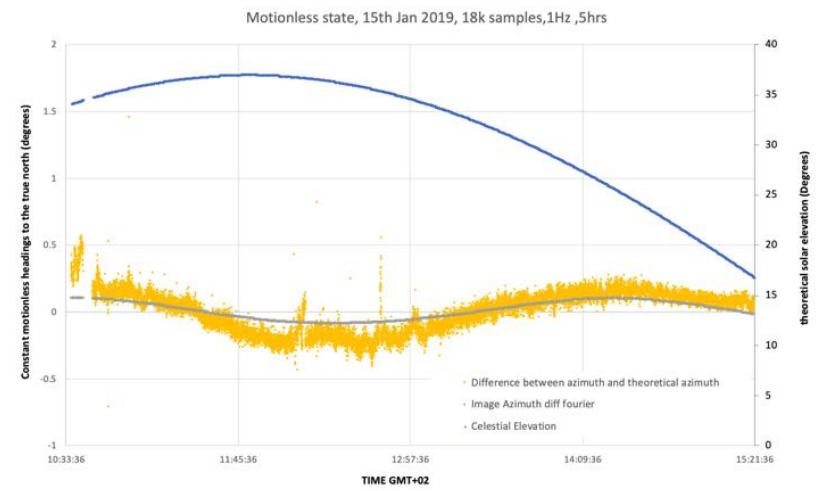

Since the UTC time was known per captured image, it was possible to define the azimuth to the true north from each image in real-time. Hence, each Yellow point in 0. represents a single sample.

Since the sensor was at a tilted position with an unknow bearings position (roll and pitch) and still the sensor was at a motionless state, it was possible by a numerical search to match the closest bearing value that agrees with the constant value of azimuth heading. The constant azimuth heading represents the sensor's motionless state. During the sensor bearings numerical search, it was assumed that the global position of the sensor was also at a motionless state.

$$
I H A=\arctan \left(\frac{\cos E l \cos (A z-B) \cos T+\sin E l \sin T}{\cos E l \sin (A z-B)}\right)+R
$$

The bearing of the sensor is a function (1) of the Image Heading Azimuth (IHA) derived from the image captured by the sensor while the solar azimuth $\mathrm{Az}$ is measured from the true north. The solar elevation $\mathrm{El}$ is measured above the horizon, while the sensor bearing B was measured from true north. The sensor's pitch position $\mathrm{T}$ is measured above the horizon and had a roll angle of $\mathrm{R}$. The numerical search has resulted in pitch value of $46.09^{\circ}$ where the roll value had a value of $3.23^{\circ}$ and the azimuth headings to the true north had a constant value of $340.30^{\circ}$, with a standard deviation of $0.14^{\circ}$.

\section{B. Finding the sensor global position of the}

Once the all sensor bearing axes where defined, roll, pitch and azimuth heading to the true north. A numerical search has been used to define the sensor global position. Using the assumption that the longitude of the sensor is known by time span between local time and UTC time. The numerical search introduced here aimed to fine the latitude position of the sensor.

The search found for each latitude line the most constant value of the sensor azimuth to the true north. This value can be represented by the standard deviation value of each azimuth to the true north at different latitude values. As much as the standard deviation is low in value, the sensor was closer to its motionless state. 
Fig. 4. A graph represents a numerical search for defining the sensor global position. The graph shows the sensor possible latitude position vs the STDV of the sensor heading to the true north, while the sensor was at motionless state.

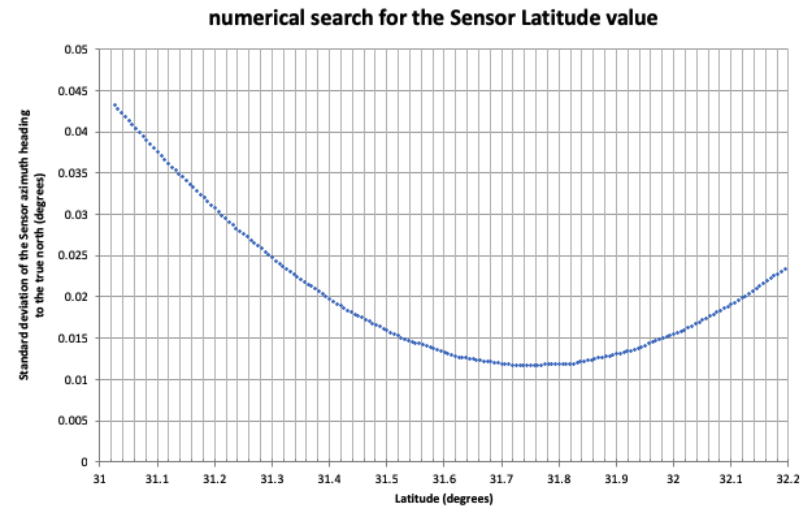

From the numerical search, the best sensor position was placed on latitude of $31.7254^{\circ}$, which is $0.1866^{\circ}$ or about $18.6 \mathrm{~km}$ south of the sensor actual global position on earth.

Since the sensor was positioned on a roof of a building, the sensor's elevation above the sea level should be taken on to account. And so, the "DIP" or height correction is needed. By applying the latitude shift of $0.1866^{\circ}$ founed earlier, it is possible to defined the sensor height using correction to the celestial elevation.

$$
\text { El at height } h=\text { El at height } 0+0.0322 \sqrt{\text { height in meters }}
$$

When converting the actual sensor global position with the latitude shift found, the sensor height above sea level should resulted in 150 meters.

\section{Conclusions}

An innovative passive optical sensor was built and tested. The sensor accurately measures its azimuth heading to the true north with standard devation of $0.18^{\circ}$ over $5 \mathrm{hrs}$ of testing at a mostionless state. The sensor also able to measure the solar elevation and its globle postion on earth using astro navigation method. The results from the test have shown that the sensor can measure celestial navigation information that can later be used for azimuth heading calibration of inertial measurement units (IMU) or magnetic-based navigation systems. And providing aid in navigate in GPS-denied environment.

The paper shows the possibilities of navigating on earth only by using the atmospheric light polarization and time keeping. The results here demonstrate the possibilities of defining the sensor bearings roll, pitch and azimuth heading to the true north. Based upon celestial navigation method, time keeping while at a motionless state, the sensor capable of finding its global position on earth.

\section{REFERENCES}

[1] S. Vorovitchik, “Apparatus and method for navigation” , US patent number US20120175496A1, 2009-09-22

[2] Roy Malkin "Understanding the Accuracy of Astro Navigation", Navigation Instructor, Navigation Training Unit HMS Collingwood, THE JOURNAL OF NAVIGATION (2014),67,63-81

[3] C. Frohlich and Glenn E. Shaw "New determination of Rayleigh scattering in the terrestrial atmosphere" in Applied Optics 19(11):1773-5 · June 1980 with 36

[4] K. J. Voss and Y. Liu, "Polarized radiance distribution measurements of skylight. I. System description and characterization,” Appl. Opt. 36(24), 6083-6094 (1997). 
[5] Huijie Zhao and Wujian Xu " A Bionic Polarization Navigation Sensor and Its Calibration Method" MDPI Sensors, 3 August 2016

[6] Miyazaki, M. Ammar, R. Kawakami, and I. Katsushi, "Estimating sunlight polarization using a fisheye lens," IPSJ Trans. Computer Vis. Appl. 1, 288-300 (2009).

[7] Y. Wang, J. Chu, R. Zhang, L. Wang, and Z. Wang, "A novel autonomous real-time position method based on polarized light and geoma gnetic field,” Sci. Rep. 5, 9725 (2015). 